\title{
Enhanced recovery pathways in thoracic surgery: the Quebec experience
}

\author{
Julien Hubert, Etienne Bourdages-Pageau, Charles Antoine Paradis Garneau, Catherine Labbé, Paula A. \\ Ugalde
}

Departments of Respirology and Thoracic Surgery, Institut Universitaire de Cardiologie et de Pneumologie de Québec, Québec, Canada

Contributions: (I) Conception and design: PA Ugalde, C Labbé; (II) Administrative support: PA Ugalde, J Hubert; (III) Provision of study materials or patients: E Bourdages-Pageau; (IV) Collection and assembly of data: CA Garneau, E Bourdages-Pageau; (V) Data analysis and interpretation: J Hubert, E Bourdages-Pageau; (VI) Manuscript writing: All authors; (VII) Final approval of manuscript: All authors.

Correspondence to: Paula A. Ugalde, MD. Division of Thoracic Surgery, Institut Universitaire de Cardiologie et de Pneumologie de Québec, 2725

Chemin Sainte-Foy, Ville de Québec, Québec G1V 4G5, Canada. Email : paula.ugalde@criucpq.ulval.ca.

Background: Canada has a universal public health system where all resources must be allocated to optimize cost-effectiveness. Rapid diagnostic assessment programs (DAPs) and enhanced recovery protocols (ERPs) may improve timeliness of care and postoperative outcomes and thus reduce costs. The use of DAPs and ERPs in lung cancer patients who undergo lobectomy via video-assisted thoracoscopic surgery (VATS) is still controversial. This study measured the time between preoperative workup and treatment with a DAP and evaluated the impact of an ERP postoperatively in patients with early-stage non-small cell lung cancer (NSCLC) who received a VATS lobectomy.

Methods: We conducted a retrospective review of patients who underwent minimally invasive lobectomy for the primary treatment of lung cancer from January 2014 through May 2017 at our institution. Timelines of care were measured. Postoperatively, the duration of chest tube drainage, length of hospital stay, and incidence of complications were noted.

Results: During the study period, 646 patients underwent VATS lobectomy for stage I or II NSCLC; of these, 384 (59\%) were assessed within the DAP. Using the DAP, the median time between the patient's first clinic visit and referral to surgery was 30.0 days [interquartile range (IQR), 21.0-40.0 days), and the median time between surgical consult and treatment was 29.0 days (IQR, 15.0-47.5 days). With the ERP, the median duration of chest drainage was 3.0 days (IQR, 2.0-6.0 days), and median hospital stay was 4.0 days (IQR, 3.0-7.0 days).

Conclusions: DAPs and ERPs have promising roles in thoracic surgical practice. A rapid DAP can expedite the care trajectory of patients with lung cancer and has allowed our institution to adhere to governmental standards for the management of lung cancer. ERPs are feasible to establish and can effectively improve clinical outcomes.

Keywords: Lung neoplasms; postoperative care; enhanced recovery; minimally invasive surgical procedures

Submitted Jan 07, 2018. Accepted for publication Jan 18, 2018.

doi: $10.21037 /$ jtd.2018.01.156

View this article at: http://dx.doi.org/10.21037/jtd.2018.01.156

\section{Introduction}

Canada's public health system is grounded in universal access to medical needs based on cost-effective quality care within federal standards. Prevention and treatment of chronic diseases, like lung cancer, is listed as a governmental focus. The timeliness of care of lung cancer patients is recognized as a quality-of-care indicator, and surgical candidates should be treated within 3 weeks of the surgical consultations. This standard meets international guidelines 
for timely care of patients and is in agreement with the findings of studies using major lung cancer databases (1-3). Unfortunately, in 2014 only $50 \%$ of lung cancer patients in Canada met this ideal timeframe for the trajectory of diagnosis and treatment (4). In contrast to Canada's standard, the British Thoracic Society recommends a delay of no more than 8 weeks between the 1st consultation with a respiratory physician and surgical resection, and that the surgery should take place within 4 weeks of placement on a surgeon's waiting list (5).

Advances in imaging techniques and diagnostic strategies have allowed better patient staging and ultimately precise treatment strategies. Rapid diagnostic assessment programs (DAPs) have been implemented in multiple centers worldwide to coordinate timely diagnostic and staging investigations, timely referral to surgery or other specialties, and to maintain contact between the patient and the referring physician. We previously demonstrated that recommended targets for patient wait times in the investigation and treatment of lung cancer can be achieved within a DAP at our center (6).

Thoracic surgery has also evolved. Minimally invasive surgery is now considered the standard approach for earlystage lung cancer patients with several proven benefits such as fewer postoperative complications, shorter hospital stays, and shorter durations of chest tube drainage (7). In fact, the feasibility, safety, effectiveness, and cost-effectiveness of minimally invasive lobectomy have all been well demonstrated as compared with open surgery (8).

Postoperative enhanced recovery protocols (ERPs) focus on improving surgical outcomes through standardized postoperative fast-track protocols, and were first described in colorectal surgery, urology, and gynecology (9-13). Surgical goals in patients with lung cancer should include a short trajectory time between diagnosis and surgery, a short hospital stay, and expedited return to daily activities. Thoracic surgeons adopted ERPs for the management of lung cancer patients treated surgically only recently $(14,15)$. The aims of this study were to measure the timeliness of care with a standardized DAP in patients with early-stage non-small cell lung cancer (NSCLC) and to evaluate the impact of an ERP in these patients.

\section{Methods}

\section{Patients}

We conducted a retrospective review of a prospectively maintained departmental database of all lung cancer surgeries performed at the Institut Universitaire de Cardiologie et de Pneumologie de Quebec (IUCPQ), a tertiary-referral and university-affiliated hospital. The database was queried for all lobectomies performed between January 2014 and May 2017. The thoracic surgery division has four board-certified thoracic surgeons, and all patients with early-stage lung cancer who were fit for surgery were approached by VATS. The Ethics Committee approved this study and waived the requirement for informed consent.

Only patients with clinical stage I or II NSCLC, according to the American Joint Committee on Cancer (AJCC) 7th edition Tumor Node Metastasis (TNM) classification (16), who were completely staged with computed tomography (CT) and positron emission tomography (PET) scanning and underwent VATS lobectomy were included in the final analysis. Invasive mediastinal staging was performed when recommended, according to the European Society of Thoracic Surgeons (ESTS) guidelines (17). Not all patients had brain imaging as part of their diagnostic staging. Video-assisted thoracoscopic surgery (VATS) lobectomy could be performed either by a uniportal or multiportal approach. Two groups of patients were identified: those who underwent a complete oncologic and clinical work-up in our institution with our standardized DAP and those who were diagnosed outside the institution and directly referred to the thoracic surgery service. The timelines were not analyzed in the latter group.

\section{$D A P$}

In September 2008, an institutional rapid DAP called "Green Files" was established as the routine trajectory for all lung cancer patients cared for at the IUCPQ. The objective was to expedite each patient's care from diagnosis to treatment. In this program, once a green file is opened, each case is analyzed by an oncology nurse navigator together with a respirologist, and all clinical tests and lung cancer staging exams are ordered. Pulmonary function tests, bronchoscopy, endobronchial ultrasound (EBUS), chest CT-scan, PET scan and brain imaging are performed within 2 to 3 weeks. Once the workup is completed, patients have a respirology consultation, and the most appropriate treatment is determined. Surgical candidates are promptly referred to the thoracic surgery clinic. When surgery is not an option because of clinical staging or comorbidities, patients have priority medical consultations with other 
specialists.

Patient education is a priority, so we dedicate a great amount of time to inform our patients about their disease, the planned surgical procedure, and the recovery period. At the thoracic surgery clinic, patients receive all necessary information about the planned surgery, and the consent form is signed. Subsequently, they meet with a surgical nurse for surgical education. All patients receive a booklet with detailed information about the surgery and the anticipated postoperative care. Patients are also referred for preoperative smoking cessation counseling and a physicaltraining program when needed. For surgery, most patients have a same-day admission, with exception of patients coming from distant regions of the province.

In this study, the timelines of care of patients with lung cancer within the DAP were examined for 3 intervals. The first one was the interval between the moment that the green file was opened until all lung cancer staging and clinical tests were performed, and patient was referred for surgery after discussion with the respirologist. The second interval was the time between the referral to the thoracic surgery department and the consult with the surgeon, and the last interval was from the surgical consult to the date of surgery.

\section{Surgical management}

VATS lobectomy was performed under general anesthesia with single-lung ventilation. The type of VATS technique was at the discretion of the surgeon; either a multiportal or uniportal approach could be used. Once the pleural space was entered, a thoracoscopic exploration was performed to confirm resectability. For lobectomy, all vascular structures were dissected separately, followed by the bronchus. Hilar and mediastinal lymphadenectomy or sampling were routinely performed. The majority of the patients did not have an epidural catheter, so they benefited from preemptive analgesia and intercostal nerve block with Marcaine $(0.25 \%)$ under direct visualization. At the end of each procedure, a 24-French chest tube was inserted for drainage.

\section{Postoperative management and discharge}

Most patients recovered in a step-down unit, though infrequently recovery in the intensive care unit was preferred. The postoperative care was via a standardized ERP for all patients (Table 1). Under the ERP, chest tube suction was not routinely used. Lower limb compression devices were removed on postoperative day 1 . The patients were mobilized 4 hours postoperatively to a chair and were encouraged to walk under supervision on the evening of the procedure. The physiotherapy department ensured that patients practiced respiratory exercises regularly and had daily walks. Family members were encouraged to help mobilize the patient. A fluid diet was started on the evening of the surgery and was progressed to a normal diet on postoperative day 1 after intravenous fluid administration was ceased.

Medications used for pain control varied according to the surgeons' preferences. In general, a combination of oral acetaminophen or nonsteroidal anti-inflammatory drugs with or without a patient-controlled analgesia (PCA) pump with hydromorphone was used. Only a minority of patients had an epidural catheter. Anesthesiologists followed all patients with epidural analgesia or PCA to optimize pain control (18-20).

\section{Discharge}

Patients were informed of the discharge 24 to 48 hours in advance. The discharge could be on the same day of chest tube removal but was more frequently the next morning. Because we are a tertiary referral lung cancer center, our patients reside throughout the province of Quebec, and discharge was occasionally delayed due to social issues. Patients were well informed before departure, and the booklet with information about postoperative care was reviewed. The first postoperative visit was scheduled for 2 weeks after discharge.

\section{Data extraction}

We searched the patients' medical records for the following general information: age, sex, tumor histology and tumor clinical and pathological stage according to the AJCC 7th edition TNM classification for malignant tumors. For patients in the DAP, we collected the date that the green file was opened, the date the patient was considered a surgical candidate and referred to thoracic surgery, the date of the first consultation in the thoracic surgery clinic, and the date of the surgery. Also, all postoperative data related to the ERP were collected including postoperative complications, the duration of chest tube drainage, the length of hospital stay and mortality outcomes.

\section{Statistical analysis}

Median and interquartile ranges (IQR) are used to 
Table 1 Enhanced recovery protocol strategies

Preoperative strategies
Preoperative visit, evaluation, and investigations with
accelerated DAP
Patient education, smoking cessation, preoperative workout
plan, and explanation of the ERP
Same-day admission
Perioperative strategies
Antibiotic and venous thromboembolism prophylaxis
Regional anesthesia as needed
Goal-directed fluid therapy
Minimally invasive surgery (VATS)
Chest drainage
Postoperative strategies
Avoidance of opiates
Avoidance of intravenous fluid overloading
Early ambulation, feeding, and physiotherapy
Rapid-recovery-directed nursing
Early removal of chest tubes
Rostoperative education

DAP, diagnostic assessment program; ERP, enhanced recovery protocol; VATS, video-assisted thoracoscopic surgery.

summarize patient characteristics and time intervals. For each patient, the number of days from opening the green file for the lung cancer investigation to surgical resection was identified. Categorical variables are reported as $\mathrm{n}(\%)$. Continuous variables are expressed as a median (IQR, 25th to 75 th percentile) depending on variable distribution. Analyses were conducted using the statistical package SAS, version 9.4 (SAS Institute Inc., Cary, NC, USA).

\section{Results}

From January 2014 to May 2017, 838 lobectomies were performed in our center. Of these 838 lobectomies, 646 were performed for the primary treatment of early-stage NSCLC (Figure 1) all by VATS. Median age of the 646 $(77 \%)$ patients who underwent VATS lobectomy was 66 years (IQR, 60-72 years). Other demographics are presented in Table 2 .

Out of the 646 VATS lobectomies, 384 patients (59\%) were diagnosed and treated at the IUCPQ using our DAP. For these patients, the median time from opening the green file until surgery was 67.0 days (IQR, 50.0-88.0 days). Median time from opening the green file until surgical referral was 30.0 days (IQR, 21.0-40.0 days), median time from referral to surgical consult was 6.0 days (IQR, 2.0-11.5 days), and median time from surgical consult to surgery was 29.0 days (IQR, $15.0-47.5$ days).

The postoperative care of all 646 patients who underwent VATS lobectomy for clinical stage I and II NSCLC, was under the guidance of our ERP. Perioperatively, median bleeding was $100 \mathrm{~mL}$ (IQR, 40-150 mL) and median operative time was 150 minutes (IQR, 123-190 minutes). The median hospital stay was 4.0 days (IQR, 3.0-7.0 days). The median duration of chest tube drainage was 3.0 days (IQR, 2.0-6.0 days). Postoperative complications were classified into pleural, cardiac, respiratory, prolonged air leak, neurogenic, and other complications (Table 3). Thirty patients $(4.6 \%)$ had pleural complications, most frequently high pleural fluid output for 5 days or more. Cardiac complications, such as arrhythmia, occurred in 83 patients (12.8\%). A prolonged air leak (classified as 5 days or more) was the most common complication and occurred in 144 patients $(22.3 \%)$. There were three deaths within 30 days of VATS $(0.5 \%)$. One patient died from septic shock. One died a sudden death after hospital discharge, and one patient who had pulmonary fibrosis died of acute respiratory failure.

\section{Discussion}

At our institution, we routinely apply a DAP and an ERP when caring for patients with lung cancer. In this study, we measured the time between preoperative workup with a DAP and treatment with VATS lobectomy in patients with early-stage NSCLC. We also evaluated the impact of an ERP postoperatively in these patients after VATS lobectomy. The rapid DAP expedited the care trajectory of our patients; however, Canadian standards for the care trajectory of patients with lung cancer were not met. Indeed, we had a median delay between acceptance on the surgical waiting list and surgery of 29 days, longer than the 21 days recommended by the Canadian Cancer Society and the Quebec Council Against Cancer (Conseil québécois de lutte contre le cancer) $(2,4)$. A number of factors may explain this delay including the referral and management of patients 


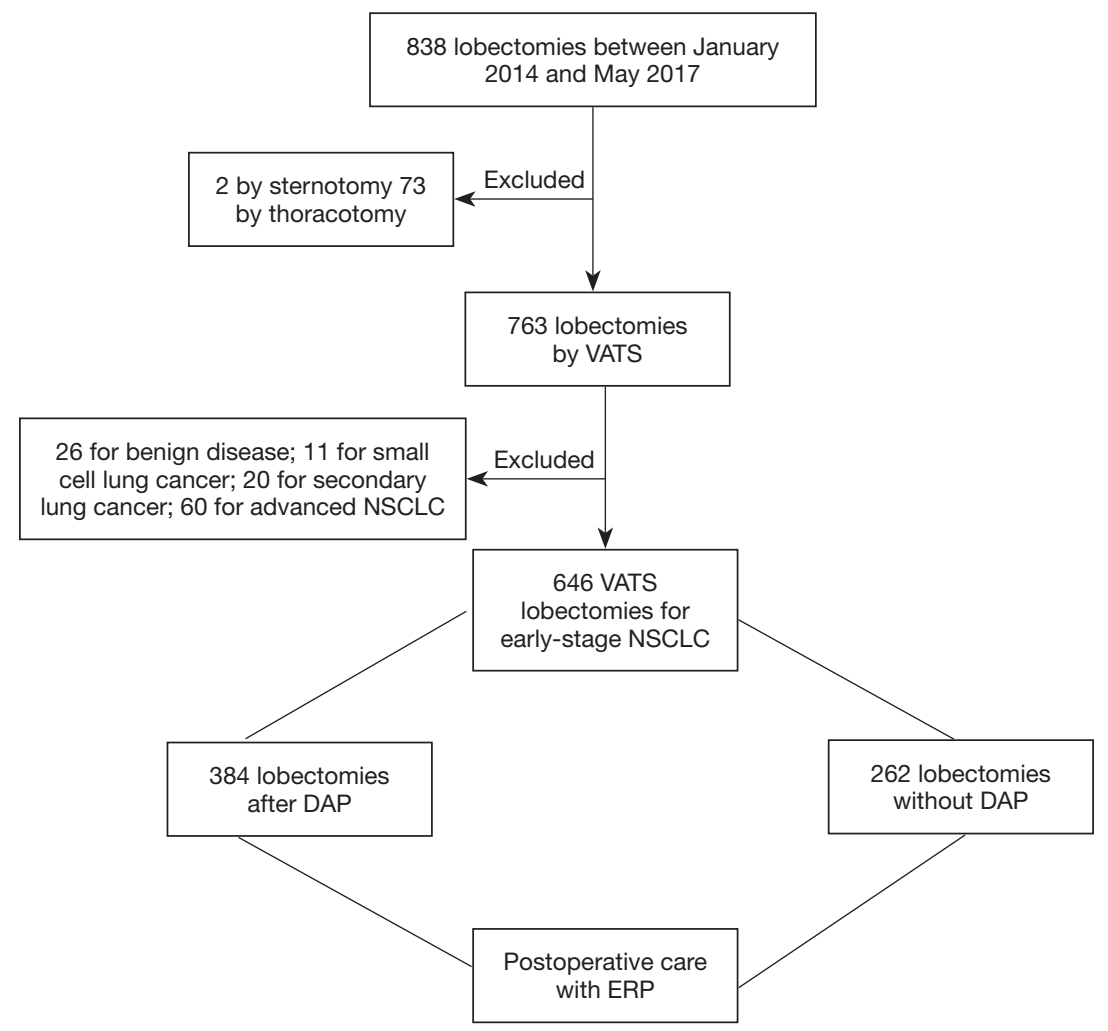

Figure 1 Flow chart of patients included and excluded from the study. DAP, diagnostic assessment program; ERP, enhanced recovery protocol; NSCLC, non-small cell lung cancer; VATS, video-assisted thoracoscopic surgery.

Table 2 Patient characteristics

\begin{tabular}{lc}
\hline Characteristics & Patients $(\mathrm{N}=646), \mathrm{n}[\%]$ \\
\hline Sex & $264[41]$ \\
Male & $382[59]$ \\
Female & \\
Histology & $475[74]$ \\
Adenocarcinoma & $125[19]$ \\
Squamous cell & $46[7]$ \\
Others & \\
Pathologic stage ${ }^{1}$ & $285[44]$ \\
IA & $181[28]$ \\
IB & $90[14]$ \\
IIA & $52[8]$ \\
IIB & $36[6]$ \\
IIIA & $0[0]$ \\
IIIB & $2[0]$ \\
IV & $w a s e d$. \\
\hline
\end{tabular}

\footnotetext{
${ }^{1}$, the 7th edition AJCC TNM classification was used
}

coming from remote regions of the province, the evolving difficulty of lung cancer cases, and the complexity of the battery of tests that need to be performed before deciding an appropriate course of treatment. When compared with the standards put forth by the British Thoracic Society (5), our waiting time from referral to surgery was comparable to their recommendation of a maximum of 4 weeks.

Patients with a diagnosis of early-stage NSCLC should be cared for with efficiency through diagnosis, staging, and treatment. Programs and strategies that reduce treatment delays lead to higher rates of surgical resection (21). Many clinicians have raised concerns that delaying surgical intervention might worsen survival, but the literature has not confirmed that there is a direct correlation between a short interval from lung cancer diagnosis to surgical treatment and survival. In 2002, Aragoneses and colleagues presented a retrospective analysis of 1,082 patients with early-stage NSCLC treated with surgical resection. The median therapeutic delay between diagnosis and surgical treatment was 35 days, and median survival was 32 months. In a multivariable analysis, they did not observe a 
Table 3 Postoperative complications

\begin{tabular}{|c|c|}
\hline Complications $^{1}$ & Patients ( $\mathrm{N}=646), \mathrm{n}(\%)$ \\
\hline Pleural & $30(4.6)$ \\
\hline Chylothorax & $8(1.2)$ \\
\hline Hemothorax & $6(0.9)$ \\
\hline Empyema & $3(0.5)$ \\
\hline High pleural effusion output ( $\geq 5$ days) & $13(2.0)$ \\
\hline Cardiac & $83(12.8)$ \\
\hline Arrhythmia & $79(12.2)$ \\
\hline Ischemic & $4(0.6)$ \\
\hline Shock & $3(0.5)$ \\
\hline Respiratory & $75(11.6)$ \\
\hline Acute respiratory distress syndrome & $6(0.9)$ \\
\hline Respiratory tract infection & $37(5.7)$ \\
\hline Atelectasis & $7(1.1)$ \\
\hline Pulmonary edema & $6(0.9)$ \\
\hline Pulmonary hypertension & $1(0.2)$ \\
\hline Pulmonary fibrosis exacerbation & $1(0.2)$ \\
\hline Respiratory failure & $23(3.6)$ \\
\hline Bronchopleural fistula & $3(0.5)$ \\
\hline Pulmonary infarction & $1(0.2)$ \\
\hline Prolonged air leak ( $\geq 5$ days) & $144(22.3)$ \\
\hline Neurogenic (Recurrent nerve palsy) & $10(1.5)$ \\
\hline Other $^{2}$ & $32(5.0)$ \\
\hline
\end{tabular}

${ }^{1}$, patients could have more than one complication; ${ }^{2}$, other complications included acute renal failure, 15 (2.3\%) patients; urinary tract infection, 7 (1.1\%) patients; digestive, $2(0.3 \%)$ patients; peritonitis, 1 (0.2\%) patient; wound infection, 1 (0.2\%) patient; cerebrospinal fluid leak, $3(0.5 \%)$ patients; other vascular complications, $3(0.5 \%)$ patients.

correlation between therapeutic delay and overall survival (22). In 2003, Quarterman and colleagues studied 84 patients with resected stage I or II NSCLC (23). Median interval between presentation and surgical treatment was 82 days. They were unable to demonstrate a negative effect of longer preoperative delays on overall survival $(\mathrm{P}=0.54)$ but argued that their confidence interval (CI) was broad and that larger sample size was necessary to reach definitive conclusions. Our median delay between diagnosis and surgical management was 67 vs. 82 days in Quaterman's study. Conversely, Yang and colleagues (3) reviewed 4,984 patients who underwent lobectomy for stage IA squamous cell carcinoma using the National Cancer Database (2006-2011). In these patients, 5-year overall survival was $58.3 \%$ (95\% CI, 56.3-60.2\%). Patients who had surgery 38 days or more after diagnosis had significantly worse 5 -year overall survival than patients who had surgery earlier [hazard ratio (HR), $1.13 ; 95 \% \mathrm{CI}, 1.02-1.25 ; \mathrm{P}=0.02]$. Further analysis is necessary to examine 5-year overall survival in our cohort and compare it with Yang's results.

Growing experience with ERPs for thoracic surgery patients confirm the role of pain control strategies and standardized postoperative care (24). ERPs typically focus on early mobilization, early chest-tube-drainage removal, and planned discharge. Scarci and colleagues recently published a retrospective study comparing 154 patients treated with an ERP and 171 patients treated in the year before introducing the ERP (14). The patients treated using the ERP showed a significantly higher number of procedures done by VATS (32.9\% VATS vs. $9.4 \%$ thoracotomy, $\mathrm{P}=0.0001)$, significantly shorter postoperative hospital stays (5.2 vs. 11.7 days, $\mathrm{P}<0.0001$ ), and higher patient satisfaction. We observed short postoperative hospital stays and an encouraging profile of postoperative complications after VATS lobectomy using our ERP.

Based on our experience and the evidence discussed above, rapidly assessing and staging patients with potentially resectable lung cancer may reduce delays prior to surgical treatment. Through minimally invasive techniques for the resection and the standard application of ERPs after surgery, lung cancer patients may benefit from fewer postoperative complications and overall superior outcomes. Even though there is not a global, organized system to diagnose and stage lung cancer and ERPs are still not widely used, our group strongly believes that this should and will become the standard. However, further analyses are necessary to evaluate if these approaches improve survival in surgical patients.

Our study has several limitations. The retrospective and descriptive qualities of the study do not reflect any comparison between patients in an ERP and those who are not. It is also a single-center study, and conclusions of our practice cannot be generalized to all centers practicing thoracic surgery. However, this study is, to our knowledge, the largest single-institution report combining a rapid DAP, minimally invasive surgical techniques, and an enhanced recovery postoperative program. 


\section{Conclusions}

In summary, ERPs should be considered the standard of care and are being applied at an increasing number of academic and non-academic institutions in growing number of surgical specialties. Thoracic surgery is following this trend, and studies detailing these experiences are getting published. Our DAP and ERP at the IUCPQ seem to present good results with acceptable diagnosis-to-treatment waiting times and postoperative hospital length of stay. Comparison between the current fast-track pathways and previous conservative management strategies at our institution has not yet been done. Cost-effectiveness and postoperative patient satisfaction also warrant further investigations.

\section{Acknowledgements}

We thank Shannon Wyszomierski for editing the manuscript.

\section{Footnote}

Conflicts of Interest: The authors have no conflicts of interest to declare.

Ethical Statement: The Ethics Committee approved this study and waived the requirement for informed consent.

\section{References}

1. Neal RD, Din NU, Hamilton W, et al. Comparison of cancer diagnostic intervals before and after implementation of NICE guidelines: analysis of data from the UK General Practice Research Database. Br J Cancer 2014;110:584-92.

2. Conseil québécois de lutte contre le cancer. Avis sur les délais dans le traitement chirurgical du cancer. Available online: http://www.msss.gouv.qc.ca/sujets/organisation/ lutte-contre-le-cancer/documents/guides-cepo-pdf/avis_ delais_traitement_cancer_2001.pdf

3. Yang CJ, Wang H, Kumar A, et al. Impact of Timing of Lobectomy on Survival for Clinical Stage IA Lung Squamous Cell Carcinoma. Chest 2017;152:1239-50.

4. Canadian Cancer Society. Canadian Cancer Statistics. Toronto: National Cancer Institute of Canada, 2015.

5. BTS recommendations to respiratory physicians for organising the care of patients with lung cancer. The Lung Cancer Working Party of the British Thoracic Society
Standards of Care Committee. Thorax 1998;53 Suppl 1:S1-8.

6. Labbé C, Anderson M, Simard S, et al. Wait times for diagnosis and treatment of lung cancer: a single-centre experience. Curr Oncol 2017;24:367-73.

7. Swanson SJ, Herndon JE 2nd, D'Amico TA, et al. Videoassisted thoracic surgery lobectomy: report of CALGB 39802--a prospective, multi-institution feasibility study. J Clin Oncol 2007;25:4993-7.

8. Paul S, Sedrakyan A, Chiu YL, et al. Outcomes after lobectomy using thoracoscopy vs thoracotomy: a comparative effectiveness analysis utilizing the Nationwide Inpatient Sample database. Eur J Cardiothorac Surg 2013;43:813-7.

9. Lassen K, Soop M, Nygren J, et al. Consensus review of optimal perioperative care in colorectal surgery: Enhanced Recovery After Surgery (ERAS) Group recommendations. Arch Surg 2009;144:961-9.

10. Varadhan KK, Neal KR, Dejong CH, et al. The enhanced recovery after surgery (ERAS) pathway for patients undergoing major elective open colorectal surgery: a meta-analysis of randomized controlled trials. Clin Nutr 2010;29:434-40.

11. Spanjersberg WR, Reurings J, Keus F, et al. Fast track surgery versus conventional recovery strategies for colorectal surgery. Cochrane Database Syst Rev 2011:CD007635.

12. Perinel J, Adham M. ERAS and pancreatic surgery: a review. Updates Surg 2016;68:253-5.

13. Coolsen MM, van Dam RM, van der Wilt AA, et al. Systematic review and meta-analysis of enhanced recovery after pancreatic surgery with particular emphasis on pancreaticoduodenectomies. World J Surg 2013;37:1909-18.

14. Scarci M, Solli P, Bedetti B. Enhanced recovery pathway for thoracic surgery in the UK. J Thorac Dis 2016;8:S78-83.

15. Schatz C. Enhanced Recovery in a Minimally Invasive Thoracic Surgery Program. AORN J 2015;102:482-92.

16. Lung. Edge S, Byrd DR, Compton CC, et al. editors. AJCC Cancer Staging Manual. 7th Edition. New York: Springer, 2010:253-70.

17. De Leyn P, Dooms C, Kuzdzal J, et al. Preoperative mediastinal lymph node staging for non-small cell lung cancer: 2014 update of the 2007 ESTS guidelines. Transl Lung Cancer Res 2014;3:225-33.

18. Davies RG, Myles PS, Graham JM. A comparison of the analgesic efficacy and side-effects of paravertebral vs epidural blockade for thoracotomy--a systematic review 
and meta-analysis of randomized trials. Br J Anaesth 2006;96:418-26.

19. Joshi GP, Bonnet F, Shah R, et al. A systematic review of randomized trials evaluating regional techniques for postthoracotomy analgesia. Anesth Analg 2008;107:1026-40.

20. Mehran RJ, Martin LW, Baker CM, et al. Pain Management in an Enhanced Recovery Pathway After Thoracic Surgical Procedures. Ann Thorac Surg 2016;102:e595-6.

21. Evans WK. Prognostic implications of treatment delays in the surgical resection of lung cancer. Thorac Surg Clin

Cite this article as: Hubert J, Bourdages-Pageau E, Garneau CA, Labbé C, Ugalde PA. Enhanced recovery pathways in thoracic surgery: the Quebec experience. J Thorac Dis 2018;10(Suppl 4):S583-S590. doi: 10.21037/jtd.2018.01.156
2013;23:225-32.

22. Aragoneses FG, Moreno N, Leon P, et al. Influence of delays on survival in the surgical treatment of bronchogenic carcinoma. Lung Cancer 2002;36:59-63.

23. Quarterman RL, McMillan A, Ratcliffe MB, et al. Effect of preoperative delay on prognosis for patients with early stage non-small cell lung cancer. J Thorac Cardiovasc Surg 2003;125:108-13; discussion 113-4.

24. Giménez-Milà M, Klein AA, Martinez G. Design and implementation of an enhanced recovery program in thoracic surgery. J Thorac Dis 2016;8:S37-45. 